\title{
Randomized clinical trial to evaluate the pathogenicity of Bibersteinia trehalosi in respiratory disease among calves
}

\author{
Christy J Hanthorn ${ }^{1}$, Reneé D Dewell ${ }^{1,2}$, Vickie L Cooper ${ }^{1}$, Timothy S Frana ${ }^{1}$, Paul J Plummer ${ }^{1,3}$, Chong Wang ${ }^{1,4}$ \\ and Grant A Dewell ${ }^{1 *}$
}

\begin{abstract}
Background: Bibersteinia trehalosi causes respiratory disease in ruminants particularly in wild and domestic sheep. Recently, there has been an increased number of $B$. trehalosi isolates obtained from diagnostic samples from bovine respiratory disease cases. This study evaluated the role of $B$. trehalosi in bovine respiratory disease using an intra-tracheal inoculation model in calves. Thirty six cross bred 2-3 month old dairy calves were inoculated intra-tracheally with either leukotoxin negative $B$. trehalosi, leukotoxin positive $B$. trehalosi isolate, Mannheimia haemolytica, a combination of leukotoxin negative $B$. trehalosi and $M$. haemolytica or negative control. Calves were euthanized and necropsy performed on day 10 of study.

Results: $B$. trehalosi inoculated calves did not have increased lung involvement compared to control calves. Additionally, B. trehalosi was only cultured once from the lungs of inoculated calves at necropsy.

Conclusions: Based on these findings B. trehalosi may not be a primary pathogen of respiratory disease in cattle. Culture of $B$. trehalosi from diagnostic submissions should not be immediately identified as a primary cause of respiratory disease.
\end{abstract}

Keywords: Bibersteinia trehalosi, Bovine respiratory disease, Calf, Pneumonia, Pasteurellosis

\section{Background}

Bibersteinia trehalosi is a known pathogen of ruminants and has been identified worldwide [1]. B. trehalosi was formerly included in a single species of Pasteurella haemolytica as biotype $\mathrm{T}[2]$. This pathogen was recently reclassified as B. trehalosi on the basis of phylogenetic studies [3]. $B$. trehalosi has been associated with systemic pasteurellosis or septicaemia in lambs [4] and pneumonia in bighorn sheep (Ovis canadensis) [5,6].

Although $B$. trehalosi infections are considered rare in cattle, the agent is occasionally isolated from bovine lungs. Diagnostic reports of severe non-responsive Bovine Respiratory Disease (BRD) outbreaks associated with multidrug resistant B. trehalosi have been documented [7]. Clinical manifestations of these strains are often associated

\footnotetext{
* Correspondence: gdewell@iastate.edu

'Department of Veterinary and Diagnostic Production Animal Medicine, College of Veterinary Medicine, lowa State University, 2237 Lloyd Vet Med, Ames, IA 50011, USA

Full list of author information is available at the end of the article
}

with multi-drug resistance and severe lung pathology. The reasons for development of these potentially highly virulent strains remain unclear. It has been hypothesized that B. trehalosi may have acquired increased pathogenicity from other bacteria. Some reports in the literature suggest that B. trehalosi and Mannheimia haemolytica can share genetic material $[8,9]$. Earlier work has shown that nucleotide diversity of lktA from B. trehalosi is minimal (0.7\%) compared to M. haemolytica (22.0\%) and were genetically different from M. haemolytica [10]. However, this report included only $B$. trehalosi isolates from ovine samples. Bovine adapted isolates of $B$. trehalosi may have acquired some genetic material from $M$. heamolytica increasing its ability to infect cattle.

Understanding the role different pathogens play in BRD is critical to understanding and effectively treating clinical cases. The primary objective of this study was to evaluate the pathogenicity of $B$. trehalosi in respiratory disease among calves using field strains of B. trehalosi. 


\section{Methods}

Two B. trehalosi isolates were identified from diagnostic submissions of bovine cases for the challenge study. Isolate identification was confirmed by $16 \mathrm{~S}$ ribosomal RNA analysis. One isolate was PCR positive for the leukotoxin (lktA) gene [11] and the other isolate was negative. The lktA gene was amplified with a $5^{\prime}$ primer lktA9 $\left(5^{\prime}\right.$-TC AAGAAGAGCTGGCAAC-3') and the $3^{\prime}$ primer lktA7 (5'-AGTGAGGGCAACTAAACC-3'). Amplification parameters were: denaturation at $94^{\circ} \mathrm{C}$ for $45 \mathrm{~s}$, annealing at $62^{\circ} \mathrm{C}$ for $45 \mathrm{~s}$, and extension at $72 \mathrm{C}$ for $2 \mathrm{~min}$. The leukotoxin-PCR-positive $B$. trehalosi isolate was cultured from the lungs, along with a Pasteurella multocida, from a feedlot calf that had been treated with antibiotics multiple times for Bovine Respiratory Disease that had fibrinosuppurative bronchopneumonia from a group of $273 \mathrm{~kg}$ Southeastern feedlot calves with $16 \%$ respiratory morbidity and $10 \%$ mortality. The leukotoxin-PCR-negative B. trehalosi isolate was cultured from the brain of a bull with multifocal, suppurative meningoencephalitis.

Thirty six 8-12 week old individually housed Holstein cross steer calves were obtained from a private calf raiser. Calves were tested for persistent infection by BVDV by immunohistochemistry prior to purchase and confirmed PI negative. Nasal swab samples were collected from each calf and submitted for bacterial culture to the diagnostic lab prior (Day -1) to inoculation. During the experimental challenge, calves were housed in a Biosecurity Level 2 facility at the ISU Livestock Infectious Disease Isolation Facility. Each calf was confined separately in raised $0.9 \times$ 1.8 meter pens that provided no opportunity for calf-tocalf contact in a room that held 12 calves at a time. Three groups of calves were utilized to accommodate room layout. Calves were provided free choice water and were fed mixed grass hay and a pre-mixed calf starter. Biosecurity procedures such as changing protective gloves and clothing between calves were employed by all research personnel working with calves. Calves were randomly assigned by a random number generator [12] to one of five treatments. Treatments were randomly assigned to 1 of 3 replicates (Table 1). Eight calves were assigned to each bacterial

\section{Table 1 Replicate and treatment group assignments}

\begin{tabular}{lccc}
\hline & Replicate 1 & Replicate 2 & Replicate 3 \\
\hline Control Calves & 1 & 1 & 2 \\
$\begin{array}{l}\text { Leukotoxin negative } \\
\begin{array}{l}\text { B. trehalosi } \\
\text { Leukotoxin postive }\end{array}\end{array}$ & 3 & 3 & 2 \\
$\begin{array}{l}\text { B. trehalosi } \\
\text { M. haemolytica }\end{array}$ & 3 & 3 & 2 \\
$\begin{array}{l}\text { M. haemolytica and } \\
\text { leukotoxin negative B. trehalosi }\end{array}$ & 2 & 3 & 3 \\
\hline Assignment of calves to treatment groups by replicate of study presented in
\end{tabular}

Assignment of calves to treatment groups by replicate of study presented in table format. challenge treatment and 4 calves to a negative control treatment.

The 5 treatment groups were:

1) Negative control (Brain Heart Infusion broth),

2) Leukotoxin negative B. trehalosi,

3) Leukotoxin positive B. trehalosi,

4) M. haemolytica

5) Combination of leukotoxin negative B. trehalosi and M. haemolytica

Prior to inoculation, each isolate was grown overnight on two blood agar plates and then transferred to a Brain Heart Infusion broth immediately prior to inoculation to obtain an estimated $2.5 \times 10^{9} \mathrm{CFU}$ of bacteria per $\mathrm{ml}$. Isolates for group 5 had an estimated $2.5 \times 10^{9} \mathrm{CFU}$ of bacteria per $\mathrm{ml}$ for both leukotoxin negative $B$. trehalosi and $M$. haemolytica for a total of $5 \times 10^{9} \mathrm{CFU}$ of bacteria per ml. A small sample of the inoculum was enumerated on blood agar plates to determine exact concentration of the inoculum. Inoculums ranged on average from $1.7 \times$ $10^{9}$ to $3.3 \times 10^{9} \mathrm{CFU}$ of bacteria per $\mathrm{ml}$ of broth. A sterile $90 \mathrm{~cm}$ number 8 French Foley catheter (MILA International) was used to inoculate each calf. The researcher administering the inoculum was masked to the treatment being administered. For each inoculation, a sterile catheter was passed intranasal to approximately the bifurcation of the trachea and $20 \mathrm{ml}$ of the inoculum was infused. Following inoculation, the calf's head was briefly elevated.

Following inoculation, calves were monitored twice daily by trained personnel masked to treatment on Days 0-9. Measurements recorded included rectal temperature, pulse rate and respiration rate. In addition each calf was clinically assessed and assigned a respiratory and depression score during twice daily monitoring. The following scoring systems were used for the study.

Respiratory scores:

0 - Normal, eyes are clear, nose is clean with no discharge, normal breathing

1 - Mild Respiratory, serous discharge from eyes and $\backslash$ or nose, slight cough

2 - Moderate Respiratory, muco-purulent discharge, cough, increased respiratory rate

3 - Severe Respiratory, excessive muco-purulent discharge, harsh cough, open mouth breathing

\section{Depression scores}

0 - Normal, cattle are bright and alert, hold their head up and readily move away from the observer

1 - Mild depression, cattle's attitude is slightly depressed but respond quickly to observer and appear normal 
2 - Moderate depression, cattle stand with head down, ears droop, abdomen lack of fill and may appear floppy, cattle move away slowly from observer

3 - Severe depression, cattle stand with head down and very reluctant to move, very noticeable gauntness of abdomen

Any calf that was assigned a respiratory and/or depression score of three was considered a candidate for euthanasia. If calf was determined to be moribund from physical exam, it was humanly euthanized. All surviving calves were euthanized on day 9 of the study by use of captive bolt followed by pithing. All euthanasias followed American Veterinary Medical Association guidelines [13].

Immediately following euthanasia, a necropsy was performed on each calf. The respiratory system was evaluated by a diagnostic pathologist. The percent abnormal lung was visually estimated for each lobe and total lung involvement calculated as a percentage of total lung as described by Jericho and Langford [14]. Samples of representative lung as determined by the pathologist were submitted ISU Veterinary Diagnostic Laboratory for routine culture and histology. For culture, samples were plated on bovine blood agar and incubated aerobically at $35^{\circ} \mathrm{C}$, sheep blood agar and incubated anaerobically at $35^{\circ} \mathrm{C}$ and tergitol-7 agar and incubated aerobically at $35^{\circ} \mathrm{C}$. All samples were incubated overnight and suspect hemolytic and non-hemolytic colonies were selected for identification by matrix-assisted laser desorption/ionization-time of flight (MALDI-TOF).

This protocol was approved by the Iowa State University Institutional Animal Care and Use Committee (\#5-127352-B) and the Institutional Biosafety Committee (\#12-I-0017-A). Personnel conducting daily monitoring, the pathologist and the statistician were blinded to calves treatment group.

\section{Statistical analysis}

Our goal was to describe the magnitude and variation of our collected measurements and assess the association of these variables with BRD and B. trehalosi. The primary outcome of interest included percent lung involvement, temperature, depression score and respiratory score. The percent abnormal lung response was analyzed using mixed effect Analysis of Variance (ANOVA) model, with Treatment as fixed effect and Group as a random effect. The remaining responses (Temperature, Depression score, and Respiratory score) were analyzed using repeated measures ANOVA model, with Treatment, time and their interaction as fixed effects and group as random effect. Comparisons among treatment groups were performed using Tukey's t-tests. SAS ${ }^{\bullet}$ Version $9.2\left(\mathrm{SAS}^{\bullet}\right.$
Institute Inc., Cary, NC, USA) was used in analyses. A p-value $<0.05$ was considered significant.

\section{Results}

Thirty five of the thirty six candidates were enrolled in the study. One calf assigned to the $M$. haemolytica group died prior to enrollment in study. Pasturella multocida was isolated from nasal swab samples from 34 of 35 calves. $M$. haemolytica was isolated from 8 of 35 calves. M. haemolytica positive nasal swab calves were fairly evenly spread across all five treatment groups (1/4 in Control group, $1 / 8$ in Leukotoxin negative B. trehalosi group, 2/8 Leukotoxin positive $B$. trehalosi group, 1/7 M. haemolytica group, 3/8 leukotoxin negative B. trehalosi and M. haemolytica combination group). Six of the 35 enrolled calves were euthanized prior to day 9 of the study. Three of the euthanized calves (3/7) in $M$. haemolytica group (days 1,2 and 2) and three calves (3/8) from the leukotoxin negative B. trehalosi and $M$. haemolytica combination treatment (days 2, 4 and 7) were euthanized according to protocol because of a clinical assessment of moribund. Overall, the mean estimated percent lung involvement was highest for the $M$. haemolytica group (49\%) followed by the mixed infection of $M$. haemolytica and leukotoxin negative $B$. trehalosi (26\%). The leukotoxin postive B. trehalosi had a mean $18 \%$ estimated lung involvement while the leukotoxin negative $B$. trehalosi mean lung involvement was estimated to be $13 \%$. The control group had a mean lung involvement estimate of $13 \%$ (Table 2). Calves with lung involvement had histologic lesions that were consistent with bronchointerstitial pneumonia (26 of 35 calves) and 20 of these calves also had evidence of pyogranulomatus pneumonia.

M. haemolytica was isolated from 5 of 7 lungs of the $M$. haemolytica inoculated group and 4 of 8 lungs from the leukotoxin negative $B$. trehalosi and $M$. haemolytica combination group. $B$. trehalosi was isolated from one of 24 lungs that were inoculated with $B$. trehalosi. Calf number 6 in the leukotoxin negative B. trehalosi and $M$. haemolytica combination inoculation treatment group was euthanized on day 2 of the study as reported above. The calf had $71.6 \%$ lung involvement and both $M$. haemolytica and a $B$. trehalosi were isolated from the lung tissue. $B$. trehalosi was not isolated from the lung tissue of any other calves that were inoculated with a $B$. trehalosi isolate.

Rectal temperature (Figure 1), depression scores (Figure 2) and respiratory scores (Figure 3) followed an expected pattern after inoculation. Overall, rectal temperatures ranged from $35.3-41.3^{\circ} \mathrm{C}$. Depression scores ranged from $0-3$ and respiratory scores ranged from $0-3$.There were occasional time periods when differences in rectal temperatures, depression score or respiratory score between the $M$. haemolytica and the B. trehalosi or 
Table 2 Percent lung involvement by treatment group

\begin{tabular}{llllll}
\hline & Control & $\begin{array}{l}\text { Leukotoxin negative } \\
\text { B. trehalosi }\end{array}$ & $\begin{array}{l}\text { Leukotoxin postive } \\
\text { B. trehalosi }\end{array}$ & M. haemolytica & $\begin{array}{l}\text { M. haemolytica and leukotoxin } \\
\text { negative B. trehalosi }\end{array}$ \\
\hline Calves & 20.4 & 0 & 36.55 & 57.25 & 71.6 \\
& 26.2 & 24.25 & 27.1 & 53.95 & 61.3 \\
& 0 & 21.7 & 28.1 & 26 & 62.85 \\
& 4.4 & 32.1 & 27.1 & 33.15 & 0 \\
& & 18.05 & 14.2 & 28.6 & 0.6 \\
& & 6.15 & 1.5 & 63.6 & 4.75 \\
Mean* & 3.6 & 11.35 & 75.1 & 6.1 \\
Adj P value** & & 1.45 & & $26.1 \mathrm{a}, \mathrm{b}$ \\
\hline
\end{tabular}

${ }^{*}$ means with different letters are statistically different $(p<0.05)$.

**Adj $p$ value of treatment from the control group.

Individual and mean lung involved in disease process as a percentage of total lung volume. Treatment group means that were statistically different from other treatment groups are indicated by different letters ( $a$ or b) in table. Adjusted p-values of treatment group from control group is reported.

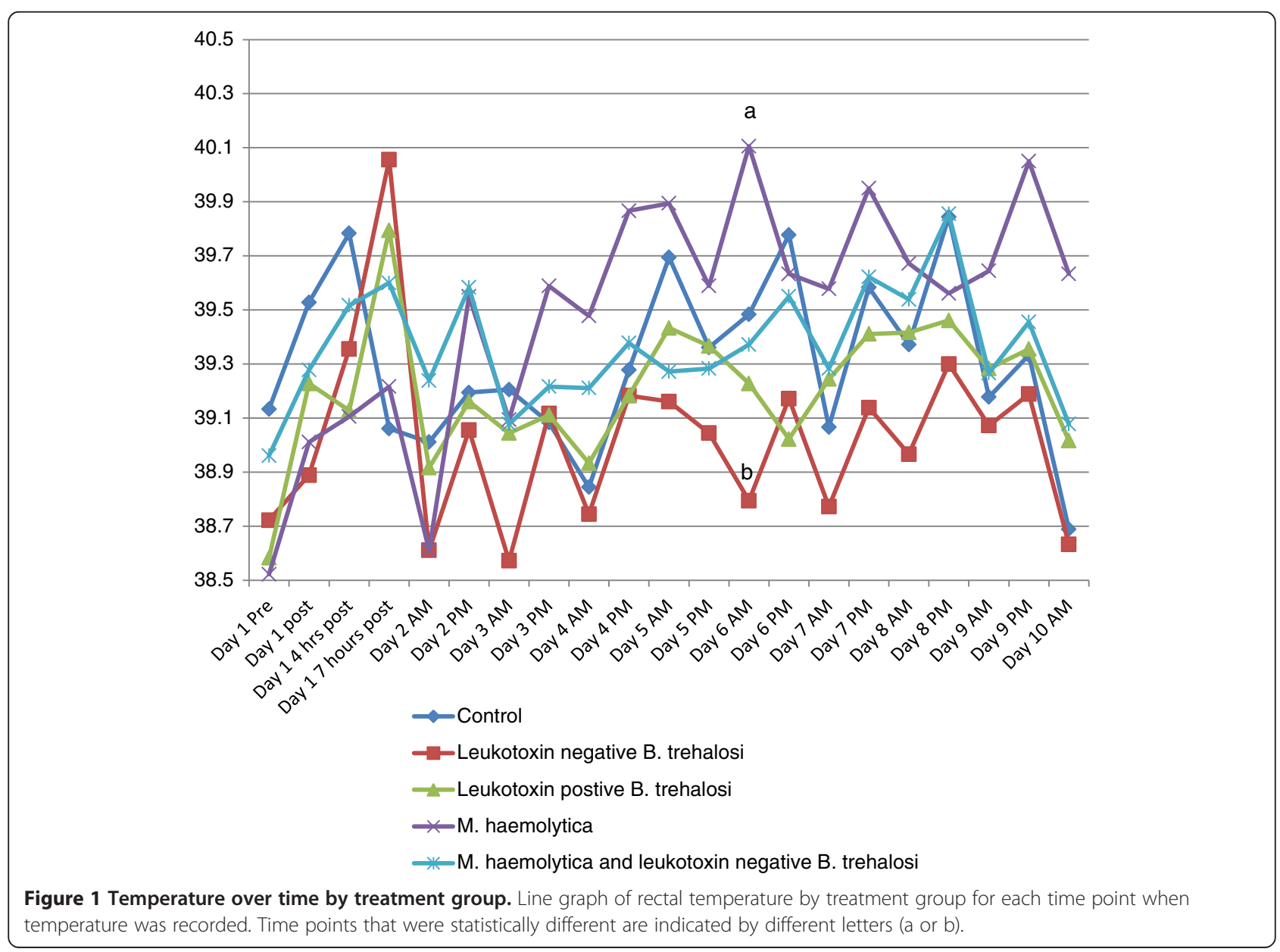




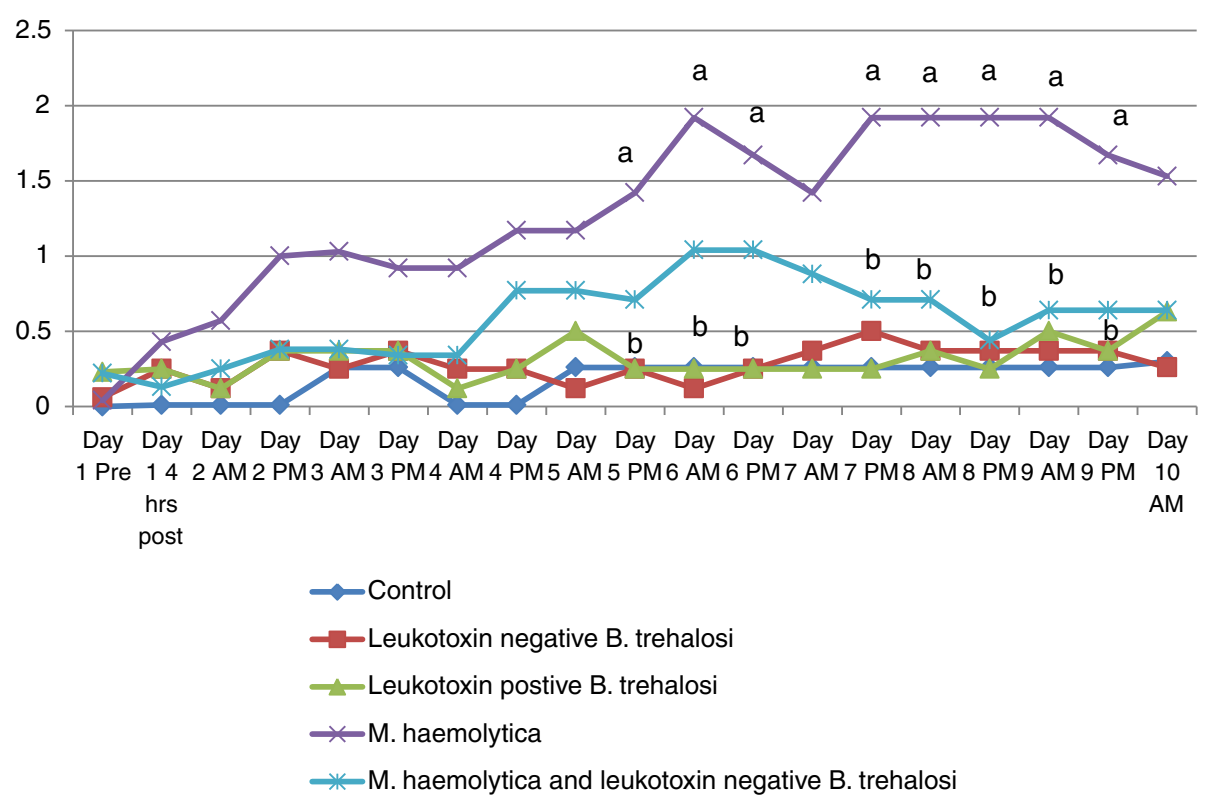

Figure 2 Depression score over time by Treatment group. Line graph of depression score by treatment group for each time point when depression score was recorded. Time points that were statistically different are indicated by different letters (a or b).

control groups were statistically significant (Figures 1 , 2 and 3$)$.

The calves from both groups inoculated with $B$. trehalosi demonstrated increased rectal temperature $\left(>39.5^{\circ} \mathrm{C}\right)$ at 7 hours after inoculation compared to calves that had been inoculated with $M$. haemolytica. Temperatures for the two B. trehalosi only treatment groups returned to normal levels $\left(37.5-39.5^{\circ} \mathrm{C}\right)$ within 12 hours. Calves in the $M$. haemolytica only inoculation group did not demonstrate increased rectal temperatures $\left(>39.5^{\circ} \mathrm{C}\right)$ on average until 24 hours following inoculation and temperatures of these calves remained elevated except for morning

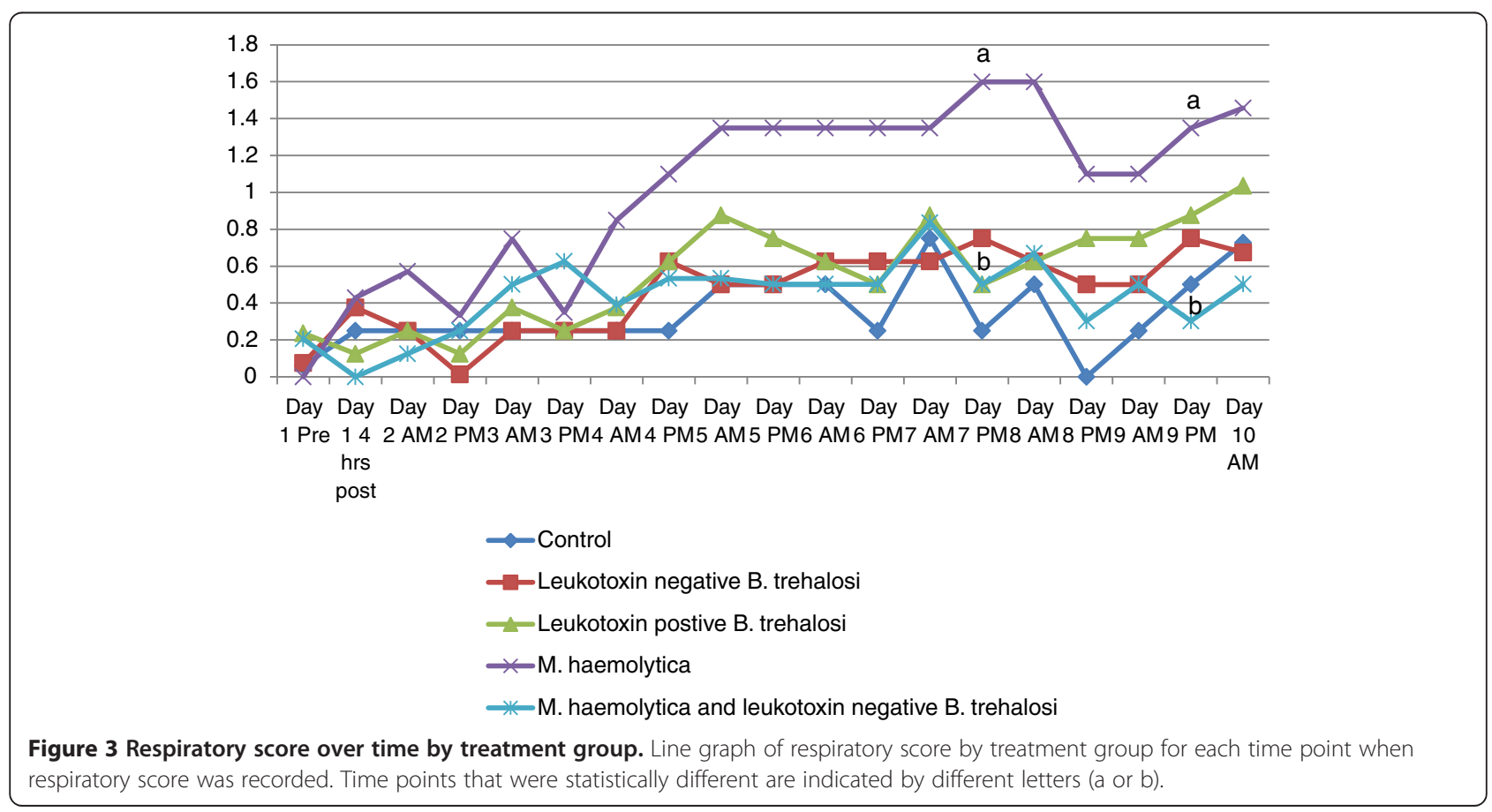


of day 2. Calves in the combined inoculation group fluctuated above and below $39.5^{\circ} \mathrm{C}$ throughout the study (Figure 1).

Depression scores (Figure 2) for the M. haemolytica inoculation group began to increase 4 hours post inoculation and remained elevated at 1 or greater throughout the study. The combined inoculation group did not have increased depression scores $(\geq 1)$ until Day 3 of the study and never reached the severity of the $M$. haemolytica only inoculation group. Neither of the B. trehalosi inoculation groups had elevated depression scores. The average depression score for the control group was 0.2. Both $B$. trehalosi only inoculation groups had an average depression score of 0.3 while the $M$. haemolytica inoculation group had an average of 1.3. The combined inoculation group had an average depression score of 0.6.Respiratory scores for the $M$. haemolytica inoculation group ranged from $0-3$ and were not consistently abnormal $(\geq 1)$ until Day 3 of the study. None of the other groups had elevated average respiratory scores (Table 3 ).

\section{Discussion}

Results of this study indicate that $B$. trehalosi isolates used in this study are not associated with significant disease in a research setting. Calves inoculated with either a leukotoxin positive (PCR positive for the leukotoxin (lktA) gene ) or leukotoxin negative isolate of $B$. trehalosi did not demonstrate increased lung involvement compared to control calves. This finding contradicts some perceptions that $B$. trehalosi is an emerging primary respiratory pathogen of cattle [7]. In contrast, this study's results are more supportive of $B$. trehalosi's role in BRD as secondary and perhaps opportunistic bacteria. Reports of $B$. trehalosi associated pneumonia are from diagnostic submissions, often without access to history of inciting causes of respiratory disease, nutritional health of calves, additional stressors or information on antimicrobial therapies. In this challenge model healthy

Table 3 Average depression and respiratory score by treatment group

\begin{tabular}{lcc}
\hline & $\begin{array}{c}\text { Average peak } \\
\text { depression score } \\
\text { (Range) }\end{array}$ & $\begin{array}{c}\text { Average peak } \\
\text { respiratory score } \\
\text { (Range) }\end{array}$ \\
\hline Control & $0.25(0-1)$ & $1(1-1)$ \\
Leukotoxin negative & $0.75(0-2)$ & $1(0-1)$ \\
B. trehalosi & $1(0-2)$ & $1.25(1-2)$ \\
Leukotoxin postive & $2(0-3)$ & $1.7(1-3)$ \\
$\begin{array}{l}\text { B. trehalosi } \\
\text { M. haemolytica }\end{array}$ & $1.25(0-3)$ & $1.25(0-3)$ \\
$\begin{array}{l}\text { M. haemolytica and } \\
\text { leukotoxin negative }\end{array}$ & & \\
B. trehalosi & & \\
\hline Average depression and & & \\
\hline
\end{tabular}

Average depression and respiratory scores over the course of the study are reported for each treatment group in table format. calves were challenged with field isolates of $B$. trehalosi and extensive lung involvement was not identified post inoculation.

The limitations of this study include the small size of the study, isolate credibility, the inoculation method and the health status of the calves prior to inoculation. The study was designed as a pilot study to evaluate the pathogenicity of B. trehalosi in cattle. The number of calves in each group was limited as much as possible to decrease the number of calves sacrificed while still providing important information. The small size of the study decreases the statistical power to identify statically significant differences even though numerically the difference between the negative control group (inoculated with broth only) and the positive control group ( $M$. haemolytica) were substantial.

The authors are unaware of any B. trehalosi isolates that have demonstrated pathogenicity in a challenge model in cattle. The B. trehalosi isolates selected for this study originated from field submissions to a diagnostic laboratory. Our approach was to use leukotoxin positive and negative $B$. trehalosi isolates that had been associated with respiratory disease and could potentially be associated with lung pathology in the study calves.

Though unlikely, it is possible that, during the inoculation procedure, the dose was inadvertently misplaced into the esophagus instead of the lungs. There are multiple bovine respiratory disease challenge models cited in the literature including trans-tracheal injection, trans-thoracic injection, bronchoscope and using a nasal-tracheal approach that this study utilized. Confirmation that the catheter was correctly placed in the trachea as opposed to the esophagus can be difficult. However, the M. haemolytica positive control group, had substantial lung involvement and a p-value of 0.07 compared to the negative control group suggesting that the inoculation technique and the challenge model was valid.

Calves in the study were conventionally reared dairy crossbred calves. Calves were purchased from a calf raiser and were considered clinically normal at the time of purchase and did not exhibit signs of respiratory disease at enrollment. However, two calves in the control group had lung lesions (20-26\% lung involvement). Thus, this pathology was presumed to have resulted from a previous infection not associated with inoculation. Evidence of lung pathology from prior respiratory disease in some control calves may confound results between treatment groups. Histologic lesions characteristic of a previous BRSV infection was seen in 17 of the calves. Since two control calves demonstrated some lung pathology, the potential difference between treatment groups and the control group was affected.

Pre-study pharyngeal swabs did not identify any calves carrying $B$. trehalosi and the calf farm did not have a 
history of $B$. trehalosi disease. Despite this, it is still possible that calves could have had exposure to B. trehalosi prior to enrollment in the project. Given the age of the calves, it is also possible that passive immunity played a role in immune response. However, there is currently not a validated serologic test for cattle to determine previous exposure to $B$. trehalosi. Therefore, it is possible that calves may have had previous unknown exposure to $B$. trehalosi that enhanced their immunological response.

It was surprising that calves inoculated with a combination of $M$. haemolytica and $B$. trehalosi tended to have numerically less lung involvement than that of $M$. haemolytica inoculation alone (difference was not significant). One would expect that mixed infections would potentiate increased lung involvement and subsequent pathology. Other research has suggested that B. trehalosi can inhibit the growth of $M$. haemolytica. Dassanayake et al. [15] reported that B. trehalosi inhibited the growth of $M$. haemolytica in co-culture when $B$. trehalosi enters the stationary phase. One possible explanation for this finding is that; when both bacteria are inoculated simultaneously, B. trehalosi may inhibit in vivo growth of $M$. haemolytica until the immune system removes $B$. trehalosi. This postulated in vivo inhibition may delay the onset of clinical signs of BRD (such as a temperature spike and decrease the subsequent severity of lung involvement.

Our goal was to determine if $B$. trehalosi is really an emerging primary BRD pathogen as has been reported or if infection occurs secondary to some other viral/bacterial insult. Cortese et al. [7] have reported peracute fatal pneumonia in healthy adult cattle attributed to $B$. trehalosi. Other, anecdotal reports concerning $B$. trehalosi and BRD, suggest that B. trehalosi is associated with severe fibinous pneumonia with consolidation (especially caudal lobes) and pleuritis that is unresponsive to antimicrobial therapy. The more minor role that B. trehalosi may play in BRD is supported by the isolation results described in this study. B. trehalosi was isolated from only one set of lungs post inoculation and in that case the sample was obtained only 3 days following inoculation from a calf that had been inoculated with both $B$. trehalosi and M. haemolytica. This finding suggests that $B$. trehalosi may not persist long term in bovine lung. The temperature response of calves inoculated with $B$. trehalosi or M. haemolytica was different $(\mathrm{p}<0.05)$ over time. The early spike and rapid return to normal in rectal temperature from the B. trehalosi inoculated calves may indicate that the immune system in the lungs may more rapidly identify and respond to a $B$. trehalosi bacterial insult compared to the M. haemolytica inoculated calves. The authors acknowledge that $B$. trehalosi isolates have been obtained from samples submitted from field cases. These "positives" suggest the occurrence of an opportunistic infection and proliferation of $B$. trehalosi after normal lung defenses have been compromised by other primary respiratory pathogens. The plausibility of $B$. trehalosi as a secondary, perhaps even an opportunistic, pathogen is a stronger argument than $B$. trehalosi as an emerging primary BRD pathogen of economic importance.

\section{Conclusion}

This pilot study suggests that B. trehalosi may not be an important primary pathogen of respiratory disease in cattle. The study's results suggest that $B$. trehalosi is likely a secondary, or perhaps opportunistic, BRD pathogen. Culture of this bacterium from diagnostic submissions should not necessarily be interpreted cited as a primary cause of respiratory disease but may still have a role as a secondary pathogen or an opportunistic invader. Clinicians and diagnosticians should temper a $B$. trehalosi culture in context of the clinical setting. Practically, identification of $B$. trehalosi as the primary isolate from a submitted lung sample may suggest a failure in identification or treatment of a major BRD pathogen such as M. haemolytica. Further challenge studies designed to assess B.trehalosi's interaction with other BRD pathogens (IBR, BVDV, Mycoplasma bovis, Histophilus somni) should be conducted. Additionally, a challenge model designed to evaluate if $B$. trehalosi, inoculated after M. haemolytica has already initiated lung damage, can lead to more severe BRD would be beneficial.

\section{Competing interests}

The authors declare that they have no competing interests.

\section{Authors' contributions}

GD and PP conceived and coordinated the experiment. $\mathrm{CH}$ and $\mathrm{RD}$ performed the experiment. VC evaluated lungs. CW conducted statistical analysis. TF helped with bacteriology guidance and draft of the manuscript. All authors read and approved the final manuscript.

\section{Acknowledgements}

The authors thank Boehringer Ingelheim Vetmedica, Inc. for their support of research through the lowa State University Foundation. Jessica Ninneman is acknowledged for her excellent laboratory assistance.

\section{Author details}

'Department of Veterinary and Diagnostic Production Animal Medicine, College of Veterinary Medicine, lowa State University, 2237 Lloyd Vet Med, Ames, IA 50011, USA. ${ }^{2}$ Center for Food Security and Public Health,

Department of Veterinary Microbiology and Preventive Medicine, College of Veterinary Medicine, lowa State University, Ames, IA 50011, USA. ${ }^{3}$ Department of Veterinary Microbiology and Preventive Medicine, College of Veterinary Medicine, lowa State University, Ames, IA 50011, USA.

${ }^{4}$ Department of Statistics, College of Liberal Arts and Sciences, lowa State University, Ames, IA 50011, USA.

\section{Received: 23 October 2013 Accepted: 11 April 2014}

Published: 18 April 2014

\section{References}

1. Franck GH: Pasteurellosis in cattle. In Pasteurella and Pasteurellosis. Edited by Adlam C, Rutter JM. London, UK: Academic Press; 1989:197-222.

2. Adlam C: The structure, function and properties of cellular and extracellular components of Pasteurella haemolytica. In Pasteurella and Pasteurellosis. Edited by Adlam C, Rutter JM. London, UK: Academic Press; 1989:75-92. 
3. Blackall PJ, Bojesen AM, Christensen H, Bisgaard M: Reclassification of [Pasteurella] trehalosi as Bibersteinia trehalosi gen. nov., comb. nov. Int J Syst Evol Microbiol 2007, 57:666-674.

4. Gilmour NJL, Gilmour JS: Pasteurellosis of sheep. In Pasteurella and Pasteurellosis. Edited by Adlam C, Rutter JM. London, UK: Academic Press; 1989:223-262.

5. Wolfe LL, Diamond B, Spraker TR, Sirochman MA, Walsh DP, Machin CM, Bade DJ, Miller MW: A bighorn sheep die-off in southern Colorado involving a Pasteurellaceae strain that may have originated from syntopic cattle. J Wild Dis 2010, 46:1262-1268.

6. Dassanayake RP, Shanthalingam S, Subramaniam R, Herndon CN, Bavananthasivam J, Haldorson GJ, Foreyt WJ, Evermann JF, Herrmann-Hoesing LM, Knowles DP, Srikumaran S: Role of Bibersteinia trehalosi, respiratory syncytial virus, and parainfluenza-3 virus in bighorn sheep pneumonia. Vet Microbiol 2013, 162:166-172

7. Cortese VS, Braun DA, Crouch D, Townsend C, Zukowski B: Case report - Peracute to acute fatal pneumonia in cattle caused by Bibersteinia trehalosi. Bov Pract 2012, 46:138-142.

8. Kelley ST, Cassirer EF, Weiser GC, Safaee S: Phylogenetic diversity of Pasteurellaceae and horizontal gene transfer of leukotoxin in wild and domestic sheep. Infect Genet Evol 2007, 7:13-23.

9. Lee I, Davies RL: Evidence for a common gene pool and frequent recombinational exchange of the tbpBA operon in Mannheimia haemolytica, Mannheimia glucosida and Bibersteinia trehalosi. Microbiology 2010, 157:123-135.

10. Davies RL, Campbell S, Whittam TS: Mosaic structure and molecular evolution of the leukotoxin operon (IktCABD) in Mannheimia (Pasteurella) haemolytica, Mannheimia glucosida, and Pasteurella trehalosi. J Bacteriol 2002, 184:266-277.

11. Davies RL, Whittam TS, Selander RK: Sequence diversity and molecular evolution of the leukotoxin (lktA) gene in bovine and ovine strains of Mannheimia (Pasteurella) haemolytica. J Bacteriol 2001, 183:1394-1404.

12. RANDOM.ORG [www.random.org].

13. AVMA: AVMA guidelines for the euthanasia of Animals: 2013 Edition. https://www.avma.org/KB/Policies/Documents/euthanasia.pdf.

14. Jericho KW, Langford EV: Aerosol vaccination of calves with pasteurella haemolytica against experimental respiratory disease. Can J Comp Med 1982, 46:287-292.

15. Dassanayake RP, Call DR, Sawant AA, Casavant NC, Weiser GC, Knowles DP, Srikumaran S: Bibersteinia trehalosi inhibits the growth of Mannheimia haemolytica by a proximity-dependent mechanism. Appl Environ Microbiol 2010, 76:1008-1013.

doi:10.1186/1746-6148-10-89

Cite this article as: Hanthorn et al:: Randomized clinical trial to evaluate the pathogenicity of Bibersteinia trehalosi in respiratory disease among calves. BMC Veterinary Research 2014 10:89.

\section{Submit your next manuscript to BioMed Central and take full advantage of:}

- Convenient online submission

- Thorough peer review

- No space constraints or color figure charges

- Immediate publication on acceptance

- Inclusion in PubMed, CAS, Scopus and Google Scholar

- Research which is freely available for redistribution 